\title{
EL VALOR DE LA PALABRA EN LA PREVENCIÓN DE LA VIOLENCIA DE GÉNERO \\ EN CONTEXTOS ESCOLARES
}

\author{
Rocío López-García-Torres \\ rocio.lopez@uchceu.es \\ Universidad CEU-Cardenal Herrera
}

\author{
Elia Saneleuterio \\ elia.saneleuterio@uv.es \\ Universitat de València
}

Recibido: 29-02-2016

Aceptado: 21-05-2016

\section{Resumen}

Los planes de prevención de la violencia en la escuela deben contemplar que esta no solo se manifiesta de manera física, sino también verbal. El lenguaje es, además, el germen del problema, especialmente en los casos de violencia de género, y en esencia aglutina parte de su solución. Reconocemos el papel fundamental de la educación en su atención y vigilancia como premisa para alcanzar la igualdad entre mujeres y hombres. Precisamente con nuestra propuesta, basada en el estudio de programas anti acoso y de prevención de conductas sexistas, consideramos diez pautas de comunicación verbal eficaces para la prevención de la violencia de género y el fomento de la igualdad, dirigidas al profesorado, que deberían asumir las políticas educativas e instituciones escolares.

Palabras clave: Prevención, acoso escolar, sexismo, estereotipos sexuales, comunicación verbal, políticas educativas, coeducación, igualdad.

\begin{abstract}
Plans to prevent violence at school should include not only physical but also verbal manifestations. Language is also the source of the problem, especially in cases of gender violence, and essentially brings together part of the solution. We recognize the underlying role of education in violence care and surveillance as a prerequisite for achieving equality between women and men. With our proposal, based on the study of anti-bullying programs and prevention of sexist behavior, we consider ten guidelines of verbal communication for the prevention of gender violence and promoting equality, aimed at teachers and recommended to political educational and educational institutions.
\end{abstract}

Keywords: Prevention, bullying, sexism, sexual stereotyping, verbal communication, educational policies, coeducation, equality. 


\section{Introducción}

Los estudios han puesto de manifiesto el papel fundamental de la escuela en el proceso y tratamiento de las diferencias entre hombres y mujeres (Rodríguez, 2003; Gregori, 2007; Aroca, Ros y Varela, 2016). En este sentido, las políticas educativas han tenido en cada época - y tienen - un papel fundamental en la construcción de los estereotipos y desigualdades entre hombres y mujeres (Butler, 2005).

Ante el fracaso de la Ley Orgánica de Medidas de Protección Integral Contra la Violencia de Género, promulgada en nuestro país el 28 de diciembre de 2004 (Bermúdez, 2015), muchos son quienes se preguntan si la clave está en la permanencia de ciertas desigualdades de género, como diferente relevancia social de los roles asumidos o implicaciones del reparto de tareas y funciones (Hurtado et al., 2015). El fenómeno social de la violencia es mucho más amplio que el problema institucional de la violencia en los centros educativos. Lo que ocurre en la institución educativa es un reflejo de lo que ocurre en la vida pública — y privada — en todos sus aspectos. Sin embargo, la literatura revisada coincide mayormente en señalar la importancia de la educación en el proceso, incluida la inteligencia emocional (Blázquez, Moreno y GarcíaBaamonde, 2009), y particularmente del papel que en ella juegan los centros escolares (Aroca, Ros y Varela, 2016).

En un informe reciente de Save the Children sobre el bullying en España, el $30 \%$ de los encuestados señalan que han sufrido violencia física y un tercio admite haber agredido a otro compañero o compañera (Sastre, 2016; Álvarez, 2016c). Según Olweus (Ortega, 1998: 39), podemos entender el maltrato entre iguales como "un comportamiento prolongado de insulto verbal, rechazo social, intimidación psicológica y/o agresividad física de unos niños hacia otros que se convierten, de esta forma, en víctimas de sus compañeros". Los estudios longitudinales demuestran que dentro de los tipos de violencia que se dan en la etapa escolar la que mayor índice de mantenimiento presenta es la que tiene que ver con conductas machistas: así, aunque no es la más frecuente en las aulas, la violencia de género entre jóvenes y adolescentes se vuelve realmente preocupante (Ortega, Ortega-Rivera y Sánchez, 2008; Fernández-Llebrez y Camas, 2012).

Un punto que merece desde el principio nuestra consideración es la importancia de los actos de habla en el tema de la prevención de la violencia, sobre todo la machista; actualmente existen iniciativas que incluso proponen legislación específica para las agresiones sexistas que con facilidad pasan desapercibidas como tales, como es el caso del piropo (Rodemann Rounsevell, 2015): en el lenguaje se manifiesta la desigualdad que, enraizada en las conciencias, genera un machismo que se traduce en actos de pertenencia o dominación, los cuales resultan violentos. Aceptando esta premisa es como nosotras proponemos, en este artículo, primar la prevención con actuaciones que tengan como base la comunicación verbal.

Con nuestra aportación, revisadas algunas de las propuestas clásicas y otras más actuales respecto de la prevención y tratamiento de la violencia machista con germen en las aulas, proponemos — entre otras pautas verbales - una comunicación entre los sexos basada en el 
respeto mutuo, en el conocimiento adecuado, en la aceptación convivencial y en el diálogo creativo. Todo ello, que ha probado ser efectivo según la bibliografía que consideramos (Gregori, 2007; Simón, 2011; Aroca, Ros y Varela, 2016, etc.), adquiere pleno sentido en contextos educativos donde el acoso escolar, en todas sus manifestaciones, sea prevenido, vigilado o atajado a tiempo.

\section{Acoso escolar y violencia de género en las aulas}

Tal y como lo define De la Encarnación (2015: 448), dentro de la noción de "acoso" cabe distinguir diversas tipologías, entre la que se encontraría el acoso escolar o bullying. Este puede ser entendido como el maltrato psicológico, verbal o físico sufrido por un alumno o alumna en el ámbito escolar, motivado por factores personales (físicos, psicológicos, de orientación o identidad sexual) o colectivos (factores étnicos, religiosos, de pertenencia a un grupo social...), de forma reiterada y a lo largo de un periodo de tiempo determinado. Puede adoptar distintas manifestaciones: la exclusión y marginación social, la agresión verbal, las vejaciones y humillaciones, la agresión física indirecta o directa, la intimidación, las amenazas o el chantaje, entre otras. Ahora bien, es importante no confundir este fenómeno con las agresiones esporádicas que puedan originarse entre el alumnado, que suponen otra tipología de violencia escolar y que, por supuesto, también deben ser atendidas aplicando las medidas educativas que el centro tenga establecidas.

Entre las características que podemos considerar definitorias del acoso escolar, destacamos, la intencionalidad, la reiteración, el desequilibrio de poder, la indefensión y personalización, el componente colectivo o grupal, el silenciamiento y la invisibilización (López-García-Torres y Saneleuterio, 2015: 425), todas ellas comunes a otras categorizaciones del acoso: independientemente del ámbito en el que se produzcan, evalúan la intencionalidad de los actos de una manera específica. Los podemos, por tanto, encontrar también entre escolares. Se trataría del acoso sexual y el acoso por razón de sexo, este último "realizado en función del sexo de una persona, esto es, con contenido sexista pero no sexual" (De la Encarnación, 2015: 449).

Próximos a estos conceptos encontramos los de violencia contra la mujer y violencia de género (De la Encarnación, 2015: 446-451). Como manifestación de la discriminación, la situación de desigualdad y las relaciones de poder del hombre sobre la mujer, esta violencia se ejerce sobre ella por el hecho de serlo. Por eso cabe distinguirla de las agresiones entre iguales. La violencia de género comprende cualquier acto de violencia basada en el género que tenga como consecuencia, o que tenga posibilidades de tener como consecuencia, perjuicio o sufrimiento en la salud física, sexual o psicológica de la mujer. Las amenazas, la presión ejercida sobre ella para forzar su voluntad o su conducta, la privación arbitraria de su libertad, 
tanto si se producen en la vida pública como privada, son comportamientos violentos por razón de género.

En López-García-Torres y Saneleuterio (2015) se adaptan a la edad escolar —y entre escolares - los tipos de violencia de género en los que coincide la mayoría de la literatura especializada, como la Declaración sobre la eliminación de la violencia contra la mujer (Naciones Unidas, 1993), proponiendo la siguiente clasificación:

a) Violencia física: cualquier acto de fuerza contra el cuerpo de una niña, con resultado o riesgo de producir lesión física o daño, especialmente si es producido por razón de ser mujer o por consideración del agresor de su superioridad sobre ella.

b) Violencia psicológica: se considera toda conducta que produzca desvalorización o sufrimiento en la niña a través de amenazas, humillaciones o vejaciones, exigencia de obediencia o sumisión, coerción, insultos, aislamiento, culpabilización o limitaciones de su ámbito de libertad. Se basa en violencia verbal y, sobre todo, en acoso relacional.

c) Violencia sexual y abusos sexuales: cualquier acto de naturaleza sexual, forzada por el agresor, mediante la fuerza o la intimidación, y no consentida por la víctima es un acto de violencia sexual, con independencia de que exista relación afectiva entre los implicados, pero también lo son, igualmente cuando estos no son consentidos, los actos comunicativos de contenido sexual.

Un cuarto tipo correspondería al de privaciones o descuido, tal y como establece el Informe mundial sobre la violencia y la salud (OMS, 1996).

$\mathrm{Al}$ abordar cuestiones relacionadas con la discriminación, las desigualdades y la violencia de género en el contexto escolar se descubren datos preocupantes, siendo necesario por ello introducir nuevas medidas y acciones que prevengan y erradiquen cualquier situación que implique discriminación, desigualdad o violencia de género, así como formas para reconocer aquellas situaciones que por su normalización o naturalización no son identificadas como actos que menoscaban la dignidad de las mujeres (López-Francés y Vázquez, 2014).

En nuestro modelo educativo sigue perviviendo más o menos explícito un patrón masculino que se valora por encima del femenino. Ello explica que ciertas conductas puedan pasar desapercibidas para personas no sensibilizadas en el tema: un desprecio hacia la inteligencia o audacia femeninas, una crítica hacia la manera de vestir de una chica o hacia lo que comenta en Internet, los celos exagerados por parte de su pareja, cualquier intento de tenerla controlada en todo momento, de saber con quién se comunica e incluso de separarla de sus amigas son solo algunos ejemplos. Puede que por sí solas no sean graves; sin embargo, conforman los primeros síntomas de la violencia de género. Y lo que es más grave es que resultan relativamente comunes entre las nuevas generaciones.

Ante ello, la educación debe incluir, como objetivo propio y legítimo, la prevención y el combate de este tipo de realidades. Además, lo cierto es que ninguna institución está más capacitada ni tiene más influencia sobre los sujetos en formación como las escuelas. En efecto, 
la educación es, sin duda, el instrumento básico para la consecución de la igualdad de oportunidades entre mujeres y hombres. La educación recibida en las instituciones educativas, desde edades tempranas, conforma uno de los pilares fundamentales de socialización y, por tanto, debe convertirse en una de las estrategias básicas para conseguir un avance significativo en la igualdad entre mujeres y hombres en todas las áreas de actuación.

Las agresiones por razón de sexo, durante la infancia, incluyen actuaciones principalmente físicas y verbales. En todo caso, suponen una parte reducida respecto al total de casos de violencia entre menores; un estudio estadístico realizado por Vicente Félix Mateo, Carmen Godoy Mesas e Ismael Martínez Ruiz (2008) en la provincia de Valencia, por ejemplo, concluye que "Respecto a la violencia sexual, aunque obtiene una baja proporción respecto al resto, constituye una gran amenaza, a la par que genera enorme angustia a todos los miembros de la sociedad en general, y de la comunidad educativa en particular" (2008: 46). Sin embargo, ello no nos debe llevar a minimizar la importancia que hay que concederle, cuando más siendo que los estudios muestran que los efectos de la violencia son diferentes según el sexo (Mercy et al., 2003; Romito y Grassi, 2007).

Si consultamos los datos de las violaciones aportados por Mercedes Bandrés (2009), las denuncias con agresor infantil son todavía menores. No son, pues, tan comunes como en otros colectivos las agresiones sexuales, encontrándonos, sin embargo, con un dato revelador: la mayoría de violaciones que se producen entre menores son colectivas. Y la mayoría de agresiones sexuales en grupo suceden entre adolescentes, apenas entre niños y mucho más frecuentemente que entre adultos.

La violencia de género en estas relaciones suele ser principalmente verbal, pero el verdadero problema radica en que, al darse entre personas en formación de su personalidad, afecta directamente a la manera en la que tanto ellos como ellas entenderán las relaciones de pareja en el futuro. Así, adolescentes a quienes no se les para los pies interiorizan el trato vejatorio en sus conductas, viéndose afectado el desarrollo de la autoestima en quienes lo consienten o sufren sin que nadie les ayude a ponerle remedio.

Además, muchos adolescentes siguen modelos de masculinidad que favorecen la violencia de género, a lo que también contribuirían ciertos deportes agresivos (Valls et al., 2008: 20). En esta rape culture o cultura de la violación se asumen ideas como que a las chicas les gustan los chicos dominantes en las relaciones, las chicas deben cuidar más que los chicos su virginidad o con quién se acuestan, ciertas maneras de vestir o de comportarse en las chicas suponen que quieren sexo, etc.

Otro problema relacionado, cuyo germen comienza en la pubertad, pero que se extiende hasta la adultez, es que ciertas conductas no se etiquetan como "agresiones" por parte de las propias víctimas, especialmente en el caso de los abusos sexuales. Ello se explica por una visión estereotipada de la violación, según la cual incluiría golpes y penetración con el pene para ser considerada como tal (cf. De la Encarnación, 2015: 540). Hay estudios (Valls et al., 2008) que demuestran que los estereotipos se reducen cuanto más diálogo hay entre las chicas sobre estos temas, pues ello ayuda a extender la tolerancia cero también hacia el control de actividades por 
parte de la pareja, la limitación de sus amistades o cualquier tipo de ridiculización. Además, igual que sucede entre las mujeres adultas, en ciertos casos se asume cierta culpabilización, tal y como señalan Rosa Valls y sus colaboradores (2008: 20). Otros factores que agravan el problema y dificultan su detección son el miedo a que llegue a la opinión pública o a posibles represalias, puesto que disuaden a las víctimas cuando se plantean denunciar su caso. El primero de los factores, además, explica a su vez que muchos casos ni siquiera sean percibidos como denunciables.

Ante esta realidad, la educación escolar no debe ser ajena a los asuntos de género. De su implicación y compromiso social dependerá la formación de ciudadanos concienciados y comprometidos con la igualdad efectiva entre hombres y mujeres y, por ende, la prevención de la violencia de género.

\section{Marco normativo para la igualdad y la prevención de la violencia escolar}

Las instituciones escolares han de contribuir al respeto de los valores de la Constitución y los derechos y libertades en ella reconocidos; asumen unos principios y valores que se fundamentan en normas legales y que se expresarán a través de su planificación y, sobre todo, en su acción. De poco serviría un discurso teórico pedagógico si las leyes educativas no contemplaran la necesidad de actuación en los centros escolares (Guijarro, 2007). Pues bien, continuando la senda marcada por la anterior ley educativa, pionera en la introducción del concepto de "coeducación", la actual Ley Orgánica 8/2013, de 9 de diciembre, para la Mejora de la Calidad Educativa (LOMCE), entre los principios de la educación, manifiesta, entre otras, las ideas de igualdad y respecto, en general y específicamente entre hombres y mujeres, como medida de prevención de la violencia de género. Respecto a sus fines, destacamos el segundo, relacionado con esta última: conseguir que se respeten los derechos y libertades fundamentales, educar en la igualdad de derechos y oportunidades entre hombres y mujeres. Y el tercero, respecto del acoso escolar en general: el ejercicio de la tolerancia y de la libertad respetando los principios democráticos de convivencia, así como la educación en la prevención y resolución pacífica de conflictos.

En cuanto al proceso y tratamiento de las diferencias entre hombres y mujeres, su papel viene siendo puesto de manifiesto parte de muchos estudios, ya que los roles se construyen a lo largo del proceso de socialización: "en pleno siglo XXI es imprescindible que esta educación se realice con una perspectiva de género, donde la equidad, entendida como una cuestión de dignidad y justicia, sea un pilar clave que aprender y vivir" (López-Francés y Vázquez, 2014: 243). En este sentido, las políticas educativas tienen un papel fundamental en la construcción de los estereotipos y desigualdades entre hombres y mujeres, y se comprometen y contribuyen a la construcción de una ciudadanía sin discriminación de las mujeres. 
Si nos fijamos en las distintas enseñanzas, para la Educación Primaria el artículo 17 de la LOMCE prevé como uno de sus objetivos desarrollar en el alumnado la capacidad de "conocer, comprender y respetar las diferentes culturas y las diferencias entre las personas, la igualdad de derechos y oportunidades de hombres y mujeres [...]". Para la Educación Secundaria Obligatoria, el objetivo en esta línea es conseguir que se valore y respete "la diferencia de sexos y la igualdad de derechos y oportunidades entre ellos", a lo que se añade el rechazo de "los estereotipos que supongan discriminación entre hombres y mujeres" (artículo 23). Los objetivos de Bachillerato y Formación Profesional también refuerzan el trabajo por la igualdad, ampliando su fomento en cuanto a derechos y oportunidades (artículos 33 y 40), así como en cuanto a pensamiento crítico hacia las desigualdades en el caso del Bachillerato.

Además, en la última década se han promulgado una serie de normas que regulan ámbitos colaterales al educativo, que ponen en marcha medidas que facilitan este avance, como es el caso de la Ley Orgánica 3/2007, de 22 de marzo, para la Igualdad Efectiva de Mujeres y Hombres, que hace mención explícita al ámbito de la educación en cuanto a los aspectos que debe tener en cuenta para la incorporación efectiva de la igualdad entre mujeres y hombres. Así, dentro del capítulo II — “Acción administrativa para la igualdad”-, se recogen aspectos tan relevantes como la garantía de "igual derecho a la educación de mujeres y hombres a través de la integración activa, en los objetivos y en las actuaciones educativas, del principio de igualdad de trato, evitando que, por comportamientos sexistas o por los estereotipos sociales asociados, se produzcan desigualdades entre mujeres y hombres". Las administraciones educativas quedan así responsabilizadas de las siguientes actuaciones, según el artículo 24 de la mencionada Ley 3/2007, llamado "Integración del principio de igualdad en la política de educación":

“a) La atención especial en los currículos y en todas las etapas educativas al principio de igualdad entre mujeres y hombres.

b) La eliminación y el rechazo de los comportamientos y contenidos sexistas y estereotipos que supongan discriminación entre mujeres y hombres, con especial consideración a ello en los libros de texto y materiales educativos.

c) La integración del estudio y aplicación del principio de igualdad en los cursos y programas para la formación inicial y permanente del profesorado.

d) La promoción de la presencia equilibrada de mujeres y hombres en los órganos de control y de gobierno de los centros docentes.

e) La cooperación con el resto de las Administraciones educativas para el desarrollo de proyectos y programas dirigidos a fomentar el conocimiento y la difusión, entre las personas de la comunidad educativa, de los principios de coeducación y de igualdad efectiva entre mujeres y hombres.

f) El establecimiento de medidas educativas destinadas al reconocimiento y enseñanza del papel de las mujeres en la Historia". 
Asimismo, y en consonancia con estudios de género (Verdú Delgado, 2014) y su importancia en la formación (Jenaro, Flores y Castaño, 2014), esta ley establece en su artículo 17 que el Gobierno aprobará periódicamente un Plan Estratégico de Igualdad de Oportunidades, que incluirá medidas para alcanzar el objetivo de igualdad entre mujeres y hombres y eliminar la discriminación por razón de sexo.

En el eje quinto del Plan de Igualdad de Oportunidades correspondiente a los años 20142016 se prevén las actuaciones destinadas a la educación, cuya ejecución se prevé en colaboración con el Ministerio de Sanidad, Servicios Sociales e Igualdad. Su objetivo estratégico, "Impulsar la igualdad de oportunidades entre mujeres y hombres a través del sistema educativo", recoge tres líneas que aglutinan las siguientes acciones (VV. AA., 2014):

a) Fomentar en los centros educativos, pero también en las familias, la realización de acciones de sensibilización y formación en cuestiones de igualdad.

b) Apoyar a las alumnas susceptibles de discriminación del tipo que sea.

c) Trabajar por la eliminación de estereotipos sexuales relacionados con estudios y profesiones, docencia o dirección de centros educativos.

\section{Prevención de la violencia de género: hacia la coeducación en la escuela}

Las profesoras Concepción Aroca Montolío, Concepción Ros Ros y Cristina Varela Portela (2016: 27) insisten en la relevancia del contexto escolar en la prevención de la violencia y la discriminación, a través de "serios y eficaces programas enmarcados en una pedagogía preventiva. Su propuesta, evaluado el problema relacional que muchas parejas adolescentes no saben resolver, se centran en las actuaciones en este contexto. Sin renunciar a ello, el programa "La máscara del amor" (Garrido y Casas, 2009) presenta unos objetivos más inmediatos, desarrollando la capacidad de reconocer cuándo una relación incluye elementos patológicos, asociados a la violencia, y da un paso más en el desarrollo de actitudes relacionadas con la igualdad de género, buscando que ellos sean capaces de identificar las señales mínimas de alarma. Lo novedoso de un programa como "La máscara del amor" es que está enfocado exclusivamente en prevenir la violencia en la pareja adolescente, con el objetivo de que "el alumnado adquiera herramientas prácticas, inmediatas y concretas para poder mantener relaciones de pareja adecuadas $\mathrm{y}$, consiguientemente, pueda rechazar las que conduzcan a pautas de dominio o de abuso aunque ya pueda estarlas experimentando (prevención secundaria)" (Garrido y Casas, 2009).

Respecto del programa de Díaz-Aguado (2002), llamado "Prevenir la violencia contra las mujeres: construyendo la igualdad", destacamos que a la hora de prevenir la violencia contra las mujeres para la construcción de la igualdad incluye temáticas como la desigualdad racial, social y de género, así como los derechos humanos de hombres y mujeres. Sus resultados mejoraron 
los conocimientos sobre las discriminaciones y la violencia de género a lo largo de la historia y desarrolló una identidad menos sexista.

Otros programas plantean la actuación de modo todavía más global, como la Guía de coeducación (VV. AA., 2008). Por su parte, las iniciativas que se centran en la prevención de la violencia, inciden en todos los tipos e intentan combatirla de manera integral en los centros (Sánchez et. al., 2001). Convencidas de que el ambiente de violencia es un caldo de cultivo para sus distintas manifestaciones, incluida la de género, y sin negar la especificidad de esta, destacamos un diseño puesto en marcha en una escuela finlandesa de Fuengirola, el programa Kiva Koulu, que es una iniciativa de la Universidad de Turku presentada en España en mayo de 2015 por el Instituto Iberoamericano de Finlandia (Hidalgo, 2016; Álvarez, 2016a). El modelo, que se ha puesto en marcha en países como Italia, Holanda, Estados Unidos y Reino Unido, además de Finlandia, se ha revelado como eficaz al lograr reducir los casos de acoso escolar (Kärnä et al., 2013), haciendo honor a su propio nombre, acrónimo de las palabras finlandesas Kiusaamista Vastaan (contra el acoso escolar).

Coinciden los expertos en que la solución al problema de la violencia y la desigualdad en las relaciones interpersonales entre hombres y mujeres, dado que se origina y conforma durante las etapas de la infancia y adolescencia, pasa por la coeducación en las escuelas. Como manifestara María del Carmen Rodríguez (2003: 58), "la escuela no puede resolver por sí sola las desigualdades que la propia sociedad general y alimenta, pero es una pieza esencial para reducirlas. En este sentido, el primer paso que ha de dar la escuela es constituirse en una institución realmente coeducativa".

Por desgracia, en las escuelas de hoy en día aún se aprecia una distancia muy grande entre el ideario normativo y la práctica coeducativa. Es urgente, pues que todas las etapas educativas estén orientadas, hacia la educación para la igualdad de oportunidades (VV. AA., 2014: 111). De ello se derivará una importante profilaxis de la violencia de género: "la coeducación también sienta las bases para erradicar actitudes generadoras de violencia de género puesto que fomenta y transmite entre el alumnado valores como la tolerancia y el respeto a la diferencia por lo que previene la aparición de conflictos en los centros escolares (VV. AA., 2008: 65).

Hace tiempo que sabemos que coeducación no es lo mismo que escuela mixta. Ni siquiera responde a una formulación extendida, según la cual "yo educo en igualdad: no hago distinciones, trato por igual a mis alumnas y a mis alumnos". El concepto se conoce mal, pues se viene insistiendo en que no es suficiente educar en igualdad, sino que hay que educar para la igualdad, esperando llegar a ella como meta cívica y objetivo educativo de primer orden, única salida para que nuestra convivencia sea saludable y realmente justa.

La coeducación es una fórmula para reequilibrar los contenidos educativos y la convivencia entre los dos sexos de forma normalizada, respetando las diferencias y manteniendo como objetivo la igualdad, para cuya consecución habrá que introducir cuantos conocimientos y habilidades sean necesarios.

El concepto de coeducación se ha ido incorporando a las políticas educativas, constituyendo un avance importante para alcanzar un modelo escolar basado en la igualdad 
entre niños y niñas, en el que se combatan la discriminación, los estereotipos sexistas y las jerarquías por motivos de género, como hemos visto que se recoge en los textos legales citados.

Sin embargo, a pesar de los esfuerzos legislativos de las últimas décadas, del progresivo cambio de mentalidad en los centros educativos y en sus prácticas, así como de la labor activa llevada a cabo en muchos ámbitos, todavía hoy perduran ciertos elementos que reproducen y perpetúan el sexismo y las diferencias entre sexos. Del mismo modo que ocurre en el resto de ámbitos sociales, en el sistema educativo en general sigue habiendo situaciones discriminatorias hacia las niñas, maestras, secretarias, limpiadoras..., respecto de sus homónimos masculinos, desmintiendo la creencia sobre la igualdad efectiva y real entre varones y mujeres como una cuestión ya alcanzada (López-Francés y Vázquez, 2014). Así lo evidencian los resultados de diferentes análisis sobre el sistema educativo español, los cuales se preguntan por las causas de esta pervivencia de diferencias que se manifiestan desfavorables a las mujeres: "El origen de esta diferencia es, sin duda, múltiple, pero es obvio que en la misma subyace, todavía, una histórica asignación de los roles" (VV. AA., 2014: 109).

Resulta indiscutible que las escuelas se encuentran ante "el desafío de dar respuestas éticas a los retos del siglo XXI": "tomarse en serio su responsabilidad social respecto al principio de igualdad entre mujeres y hombres es esencial” (López-Francés y Vázquez, 2014: 249-250). Estamos de acuerdo con los expertos en la materia cuando afirman que es imprescindible coeducar para construir una ciudadanía en igualdad.

Dado que en la escuela la idea de igualdad de derechos y oportunidades ha hecho bastante recorrido, es un ámbito propicio para sembrar la convicción acerca de la necesidad de cooperación entre los sexos. Ha de adoptarse para ello, además del valor de la igualdad como discurso, la práctica de la misma, adaptando sus modos y también sus contenidos hacia una intervención decididamente coeducativa, con enfoque de género, no sexista. Se trata de impulsar proyectos coeducativos que sirvan para contribuir a superar las limitaciones estereotipadas de roles y permitan un desarrollo más equilibrado y libre de la personalidad, ayudando a construir relaciones entre los sexos basadas en el respeto y la corresponsabilidad e impulsando la participación de hombres y mujeres en todos los espacios de la sociedad

En este sentido, y siguiendo a María Elena Simón (2011: 193-194), mostramos las siguientes consideraciones que deben ser tenidas en cuenta. Lo primero es que no trabajamos por la coeducación si suponemos el trato igual como habitual o conseguido, y la igualdad de oportunidades como indiscutiblemente generalizada. No hemos de olvidar que los roles sexuales están adquiridos social y familiarmente, así como normalizados por la costumbre; asimismo, no podemos considerar los comportamientos como naturales de cada sexo. Por supuesto que habrá diferencias entre sexos, pero no podemos permitir que estas se conviertan en desigualdades. Establecer un modelo androcéntrico y animar a las niñas a alcanzarlo no es posibilitarles la igualdad; es decir, hacer que las niñas se adapten a lo masculino no es coeducación: jamás es mejor ni deseable. Complementariamente, no podemos bajar la guardia ni pasar por alto micromachismos cotidianos: la verdadera coeducación debe concederles la importancia que se merecen, jamás debe considerarlos bromas o cosas de chicos. Así, consentir 
que se hagan deducciones generalizadoras — "las chicas no pueden...", "los chicos no deben..."- es fomentar las barreras entre sexos y la diferenciación de roles, lo que equivale a consentir la existencia de obstáculos mentales en el camino hacia la igualdad. En suma, no debemos reforzar estereotipos sociosexuales, suponiendo la división sexual del trabajo y la familia, ni siquiera alimentar fantasías o quimeras de género, aunque parezcan inofensivas, del tipo Bella Durmiente o Supermán.

Hasta aquí, hemos formulado lo que no es coeducación, en negativo. Pero no caer en los errores enumerados no es suficiente para alcanzar la coeducación (González-Gil y MartínPastor, 2014); además hay que trabajar en positivo (Simón, 2011): en primer lugar, debemos nombrar siempre a las niñas, chicas o mujeres de forma justa y equivalente. Respecto a los modelos no estereotipados relacionales, familiares, laborales, de mujeres y de hombres, nuestro discurso siempre debe legitimarlos, a ser posible de forma expresa. Por otro lado, debemos aprovechar cualquier contenido curricular para introducir la presencia y la obra de las mujeres, según la época y cultura que estemos tratando: ninguna de ellas carece de mujeres insignes o cuya labor no merezca la pena ser divulgada. Ante la presencia, en ideas traídas de la familia o presentes en los medios de comunicación, de prejuicios y suposiciones sociales y familiares sobre las niñas y los niños, la labor docente debe contrarrestar explícitamente esos modelos. Asimismo, es coeducación poner a cada alumno o alumna, independientemente de su sexo, frente a sus capacidades personales, para que elijan opciones vitales o profesionales sin prejuicios. $\mathrm{Y}$ en las relaciones amistosas y grupos sociales que se van creando entre nuestro alumnado, debemos favorecer la cooperación entre iguales evitando exclusiones, competitividad o rivalidad excesivas en reductos masculinizados o feminizados en exceso. Por último, la coeducación requiere crear un estado de opinión negativa respecto al machismo, el sexismo o la discriminación sexual. Pero para ello, siguiendo estudios recientes (Diego y González, 2014; Contreras y Trujillo, 2014) es necesario incluir un enfoque adecuado de la educación sexual en la escuela.

En todo caso, la coeducación para la igualdad se tendrá que realizar en múltiples ámbitos escolares de actuación, para poder ir transformándolos poco a poco. Siguiendo a M. ${ }^{a}$ Elena Simón (2011) y adaptando alguna de sus sugerencias, estos podrían quedar enmarcados en cinco ámbitos, que serían los siguientes:

a) Relaciones entre chicas y chicos, profesoras y profesores, madres y padres, personal no docente y todas las interacciones cruzadas que se producen.

b) Saberes formales, incluyendo la obra humana de las mujeres, la educación sexual y para la autonomía personal y el cuidado a uno mismo y hacia el prójimo.

c) Materiales didácticos y metodologías de aprendizaje.

d) Comunicación y lenguajes para la igualdad.

e) Prácticas relacionadas con cargos e instancias de responsabilidad, poder y decisión.

f) Formación para la igualdad y fomento de una nueva cultura escolar, que pueda ir desterrando el machismo, el sexismo y el androcentrismo, identificándolos previamente. 


\section{Decálogo de comunicación verbal para la prevención de la violencia de género y la promoción de la igualdad en el marco escolar}

Combinando los consejos y metodologías de todos los estudios e iniciativas a los que hemos hecho referencia, el resultado de nuestra investigación establece una serie de pautas basadas en la actuación verbal, dado que es de donde surge parte del problema y en la que se basa la acción docente cotidiana. No podemos bloquearnos pensando que es muy complicado. Podría sugestionarnos la idea de que no debería ser mucho más difícil que incorporar las tecnologías de la información y la comunicación (TIC) o el bilingüismo a las prácticas docentes para poder ir transformando poco a poco el ámbito educativo.

A partir de los programas analizados, podemos afirmar que los planes antiacoso incluyen regulaciones específicas del tiempo y la observación, como el aumento y control del nivel de alerta del profesorado, las estrategias de eficacia de la detección, la reducción del tiempo de recreo, con la subsiguiente mayor frecuencia de las pausas, contratación de guardianes, etc. Sin embargo, y aun reconociendo que todo lo mencionado es efectivo, en este artículo nos centramos en aquellas actuaciones que tienen como base el lenguaje, conscientes de que la naturaleza de un problema debe estar en la naturaleza de su solución.

En concreto el profesorado, por su parte, ¿a qué debe atender? El profesorado desempeña el papel más importante dentro del modelo de escuela coeducativa puesto que son quienes están en contacto directo con los chicos y las chicas. Tienen la capacidad de transmitir pedagógicamente, además de los conocimientos del currículo, los valores para favorecer la igualdad entre hombres y mujeres (VV. AA., 2008: 60).

Estaremos de acuerdo en que los maestros y profesores son, en efecto, el principal motor del cambio en el seno de las instituciones educativas (Rodríguez, 2003). Es más, las propias acciones de los docentes, en cuanto agentes socializadores, son interpretadas por el alumnado y también influyen en la configuración de esa sensibilidad hacia la igualdad.

Exponemos, pues, a continuación, las pautas de actuación basadas en el lenguaje verbal, por las que todo docente debería velar si quiere sumarse al cambio de modelo educativo que crea en la seguridad, igualdad y respeto personal, como premisa para un clima educativo en el que transmitir conocimientos y desarrollar competencias en cada una de las áreas del currículo.

\subsection{Conoce a tus alumnos y vela por sus derechos verbales}

Para el conocimiento hay que mostrar interés por la vida privada de los estudiantes, por sus intereses y sistema de valores. Es fundamental estar abiertos al diálogo, prever tiempo para desarrollar una relación que, dentro de los límites que corresponden a la relación con el alumnado, nos permita detectar cuándo alguien no está a gusto por algún motivo, así como percibir señales de que algo no funciona como siempre. Tengamos en cuenta que el maltrato psicológico es mucho más sutil que el verbal o físico, porque puede no ir acompañado de vejaciones evidentes ni gestos agresivos. 
Además del derecho al respeto de las propias convicciones y del derecho a la integridad y la dignidad personal, hemos de velar por el derecho a la libertad de expresión. Todo estudiante tiene derecho a expresar sus opiniones verbalmente, de manera oral o escrita, individual o colectiva, teniendo en cuenta los cauces que el centro establezca y siempre dentro de los límites previstos en la legislación vigente, es decir, respetando los derechos de las personas a quienes afecten sus palabras.

\subsection{Sé vigía del lenguaje}

Normalmente hay insultos leves a los que no se suele dar importancia. Sin embargo, algo que nos han enseñado las escuelas antiacoso con programas como Kiva es que no se puede dejar pasar ni un "tonto" (Álvarez, 2016a). Puede que parezca exagerado, pero si somos capaces de acostumbrarnos a lenguajes relajados, e incluso si nuestros oídos llegan a asimilar como parte de la cotidianidad ciertas palabras malsonantes, debemos pensar que también lo contrario es perfectamente posible. Este mismo celo debe aplicarse al uso machista del lenguaje: un profesorado concienciado transmite esta consciencia en su labor educativa.

Resulta necesario detenernos en las siguientes evidencias, que hay que tener en cuenta para la efectividad de los planes de prevención, a la hora de su diseño: quien agrede verbalmente no tiene por qué sobrepasar la barrera de la herida física. Frecuentemente, empero, quien agrede físicamente, también lo hace verbalmente.

Asimismo, el educador debe ser un centinela de lo que los menores consumen, aunque sea en situaciones de juegos o en argumentos ficticios. En este sentido, no debemos dudar de que resulta necesario velar por evitar la normalización de las agresiones en la fícción. Recientemente han saltado a la opinión pública casos muy llamativos de enaltecimiento del terrorismo y la violencia (Barroso, 2016), pero otras veces nuestros menores están expuestos a escenas no aptas para ellos con el desconocimiento de los adultos que muchas veces los dejan sin supervisión delante de la televisión

Los docentes no pueden vigilar este aspecto, pero sí pueden orientar a los padres y madres. Y en lo que les compete, sí pueden velar por la adecuación de las obras contenidas en la biblioteca de aula (Cabrera García-Ochoa y Martínez-Bello, 2014), así como tomar el relevo ante cierta publicidad (Hidalgo-Marí, 2015), cuando se visionan películas en determinados momentos escolares o cuando se asiste a representaciones con los alumnos y alumnas.

Tu propio lenguaje debe ser ejemplo de respeto absoluto. Piensa que forma parte de tu trabajo controlar tanto el tono de voz en situaciones tensas como despejar tus intervenciones de toda intención hiriente, además del ya comentado uso no sexista del lenguaje, dando paso a una forma de nombrar incluyente, justa y adecuada a las nuevas realidades (Simón, 2011). 


\subsection{Da retroalimentación tras un conflicto}

Si los niños pueden ser muy crueles con otros niños, promoviendo la insolidaridad y la exclusión, la labor docente debe compensarlo conceptual y actitudinalmente poniendo de relieve que se trata de conductas detestables, que no tienen justificación alguna, haciendo hincapié mediante ejemplos reales en las consecuencias que estas dinámicas conllevan para el agresor y la víctima - especialmente en las menos evidentes para los niños, como que el agresor termina sintiéndose muy mal por haber hecho daño, sentimiento que se debe asimismo trabajar en los casos que pudieran darse en la escuela-. En general, se pondrá de manifiesto que la agresión nunca constituye una acción eficaz para manejar la situación o lograr objetivos.

Sin embargo, no todo deben ser actitudes punitivas, pues pueden resultar contraproducentes si generan resentimiento. Habrá momentos, cuando se trate de burlas, de bromas o de frases irónicas, en que una estrategia puede ser canalizada, para que no sean origen de algo más grave, hacia la idea de "me río contigo, no de ti". Actitudes de risa sana fomentan el buen clima, siempre respetando la premisa de que los chistes no sean ofensivos, groseros o puedan herir sensibilidades.

En todo caso, no dejes pasar un altercado entre compañeros para comentar lo sucedido con el grupo clase. De hecho, momentos como estos pueden justificar, por ejemplo, la impartición de una de las sesiones del curso de formación específica en materia de violencia escolar y machismo. Sin embargo, no debemos confundirnos: no se trata de depender de que ocurra un caso de maltrato para tomar la iniciativa instructora, pero sí se pueden aprovechar casos reales, cercanos o no, para dar esa retroalimentación crítica o introducir ciertos temas que no deben eludirse en la escuela. Se trata precisamente de la diferencia entre la prevención primaria y la secundaria que señalan María del Mar Muñoz Prieto y María Sandra Fragueiro Barreiro (2013: 43); la terciaria supone un paso más, al ser la que ocurre "cuando nos encontramos ante situaciones violentas ya consolidadas. Las actuaciones están diseñadas para remediar el problema, disminuir la duración y minimizar los efectos negativos y perjudiciales para el alumnado" (Muñoz Prieto y Fragueiro Barreiro, 2013: 43-44).

\subsection{Fomenta el diálogo}

Muchos de los programas revisados incluyen formación específica en la detección de las señales de alarma. Si bien no discutimos que esta capacidad debe ser desarrollada para poder ser ejercida de manera autónoma, no debemos olvidar insistir en la importancia del diálogo: todo signo percibido, en primera persona o como testigo, debe ser susceptible de convertirse en materia de conversación con otras personas que nos ayuden a definirlo y atajarlo. Estas personas pueden ser familiares, amigos o incluso el personal del centro educativo. Lo importante es poder contar con interlocutores adecuados. 
Asimismo, el diálogo entre las propias personas que protagonizan el altercado, es decir, entre los agresores y las víctimas, bien enfocado también funciona como bálsamo para ambos y como prevención o suavizamiento de futuros episodios.

En general, para favorecer la supresión de estas tendencias es importante promover en los escolares una actitud crítica y reflexiva respecto a la violencia que les rodea, enseñándoles a rechazar la violencia en cualquiera de sus manifestaciones. Y la intervención que se programe para prevenir o abordar los problemas de violencia entre iguales en los centros educativos no debe estar dirigida exclusivamente a las víctimas, sino también a los agresores y a los espectadores porque todos están implicados y para todos tiene consecuencias negativas.

En esta línea, que el profesorado fomente un buen clima en el aula a través del diálogo parece ser un factor de protección de la violencia en la escuela, y el consenso de normas en el aula podría ser una de las vías para conseguirlo (Povedano et. al., 2015).

Asimismo, fomentar el trabajo y comunicación en grupo ha demostrado ser efectivo en la reducción de conductas violentas, porque ayuda a establecer pautas de cohesión entre los miembros. Obliga al diálogo, al acuerdo y al consenso. Todos son importantes y aprenden a apoyarse mutuamente en beneficio común. No es casualidad que el lema del programa finlandés Kiva, que está revolucionando los métodos antibullying, sea precisamente "Let's make it together!".

La promoción de las tomas de decisiones democráticas, la preeminencia de las formas pacíficas en la resolución de conflictos, haciendo frente a las tensiones sociales mediante la utilización del diálogo y la negociación, son medidas imprescindibles para la prevención de la violencia escolar. Hay que educar en el respeto y la convivencia armoniosa, orientada a evitar la generación de personalidades tendentes tanto a un perfil de agresores como de víctimas.

\subsection{Prima la comunicación inmediata}

Esperar a que el problema se complique es un error que puede tener consecuencias lamentables, por eso es necesario que otros profesores o profesoras conozcan cuanto antes el problema en cuestión, e incluso que se informe a la dirección del centro. Actualmente, además, las leyes están pasando a considerar que ser testigo de un caso de acoso y no comunicarlo es motivo de sanción grave (Álvarez, 2016b). Dice al respecto la escritora Rosa Montero (2016): "La verdadera culpa del acoso escolar está en los adultos perezosos y cómplices, en el profesorado, los padres, las instituciones". Debemos, pues, pensar, que no nos compete a nosotros juzgar la gravedad de un asunto: hemos de dar constancia del mismo, para no ser cómplices de los agresores con nuestro silencio.

En este sentido, no demores el intercambio de impresiones con las familias. Los especialistas en cuestiones de prevención de violencia escolar incluyen, entre sus medidas recomendadas, el establecimiento de comunicación fluida escuela-hogar. Efectivamente así se recoge en muchos proyectos antiacoso, como por ejemplo el método finlandés Kiva, revolucionario en las sociedades occidentales, como hemos comentado. Así pues, según sus 
directrices, es necesario hablar, desde los primeros signos, con los padres, madres o personas que ejerzan la tutoría legal de los menores implicados en un altercado. Ellos deben tener a su disposición guías y tutorías para poder hacer frente a este tipo de situaciones. Una de las más populares y efectivas en la actualidad es precisamente KiVa Antibullying Program. Parents' Guide, disponible en la red de manera gratuita (Kaukiainen y Salmivalli, 2009).

Hemos de tener en cuenta que los programas más efectivos prevén soluciones grupales a los problemas de violencia o acoso, dado que el bullying suele tener su origen también en grupo: desde los planes estatales hasta las familias, pasando por los educadores, todos han de colaborar en un diálogo constructivo con acosadores y acosados, también en casos de violencia de género.

\subsection{Aprovecha la sensibilización mediante la literatura o el arte}

Recientemente, en un análisis del fracaso de la Ley Orgánica 1/2004, Silvia Bermúdez (2015) se plantea el papel de las manifestaciones culturales, como el cine o la literatura en la tarea de tomar responsabilidad colectiva de esta lacra social.

La escenificación del amor tóxico, aunque no sea específicamente en películas sobre adolescentes, ayuda en el proceso de identificación y rechazo, desde los primeros síntomas, del problema. El hecho de asistir como espectadores no impide la identificación por parte del receptor con la problemática de los personajes, pero precisamente la condición de espectador, y sobre todo el planteamiento didáctico desde las aulas o el cine fórum, posibilitan la distancia crítica que se requiere para una asimilación de sus peligros e implicaciones. Películas que se usan frecuentemente para tratar estos temas son el cortometraje, en forma de falso documental, Amores que matan (2000) o la galardonada Te doy mis ojos (2003), ambas de Icíar Bollaín. Otras asimismo útiles, podrían ser Solas (1999), de Benito Zambrano; En la puta vida (2001), de Beatriz Flores Silva; Sólo mía (2001), de Javier Balaguer; Princesas (2005), de Fernando León de Aranoa.

También se han intentado experiencias didácticas que parten de la literatura, con novelas como Algún amor que no mate (1996), de Dulce Chacón, La mujer del héroe (2005), de Consuelo Alcalá, e incluso el poemario de Ana Romaní Love me tender. 24 pezas mínimas para una caixa de música (2005), entre muchos otros que se pueden citar. Sea cual sea, lo importante es no perder de vista la utilidad de la literatura para estos propósitos (Aroca Montolío, Ros Ros y Varela Portela, 2016).

\subsection{Descubre nuevas metodologías docentes}

En los métodos de enseñanza y aprendizaje hay mucho camino por hacer, pero también mucho potencial explorable: en nuestro horizonte hemos de favorecer aprendizajes significativos por medio de la observación, la crítica y la indagación, que ayuden a deconstruir falacias, falsas creencias, mitos e ideas previas erróneas o discriminatorias (Simón, 2011). 
Muy relacionada con la educación lingüística y literaria, la metodología denominada "tertulias literarias dialógicas" establece objetivos formativos que aúnan objetivos de formación estrictamente literaria con horizontes de animación a la lectura y promoción de espíritu crítico, todo ello mediante la lectura compartida y el debate moderado entre los propios estudiantes, lectores e interpretadores de las obras propuestas. En este sentido, no deberíamos desaprovechar las ocasiones para acercarnos críticamente a concepciones que quedan entrelíneas y que justifican ciertas conductas y sistemas de valores, o discutir de la imagen de la mujer de ciertas obras (Saneleuterio, 2010).

\subsection{Lleva un registro detallado}

Los casos de violencia o maltrato verbal deben quedar archivados, para su seguimiento e incluso para su estudio en aras de acciones preventivas. Este puede ser un granito de arena hacia un registro común estatal que centralice los casos y que permita observar pautas de comportamiento y predecir riesgos. De hecho, desde el Ministerio, el borrador del plan de convivencia promete poner en marcha antes de junio de 2016 un registro estatal sobre casos de acoso escolar y otros casos de violencia en las aulas.

Asimismo, algunas comunidades autónomas acaban de poner en marcha iniciativas relacionadas, como el segundo Plan de Igualdad de Género en la Educación 2016-2021 aprobado por el Consejo de Gobierno de la Junta de Andalucía durante el mes de febrero de 2016. Entre las novedades concretas de esta Comunidad Autónoma destaca también la creación de la Red Andaluza de Centros Igualitarios y Coeducativos, que visibilizará y apoyará a los colegios e institutos que lideren experiencias en este ámbito.

\subsection{Colabora en la redacción y cumplimiento del proyecto educativo y plan de igualdad}

Para llevar a la práctica la coeducación en la escuela una de las premisas es la existencia de un Proyecto Educativo de Centro en el que la coeducación esté planificada, coordinada y tenga en cuenta los recursos disponibles para optar por la vía más adecuada para su desarrollo (VV. AA., 2008: 19).

"En suma, la coeducación se plantea desde una doble perspectiva: debe asegurar una práctica educativa que no sea discriminatoria para con el género femenino y, además, debe hacer visibles y extender a toda la población escolar aquellos valores propios de la cultura femenina que tradicionalmente han sido objeto de una discriminación en el currículum y en la vida cotidiana de las escuelas. Estos son los dos puntos básicos sobre los que ha de pivotar un adecuado proyecto educativo" (Rodríguez, 2003: 59). 
De acuerdo con la estrategia aprobada, los centros deberán elaborar planes de igualdad específicos e incorporar sus objetivos en los de convivencia y los de orientación y acción tutorial, así como en las programaciones didácticas. El plan de igualdad específico de cada centro educativo, obligatorio en algunas comunidades autónomas, es un elemento valioso en la prevención y lucha contra el sexismo y la violencia escolar. Si no existe, tú puedes tomar la iniciativa. Además de la implementación de medidas de prevención, una de las manifestaciones es la promoción de talleres específicos para el profesorado y los equipos de apoyo psicopedagógico sobre las señales - muy sutiles en ocasiones-, que permitan una detección precoz de situaciones de violencia escolar y, principalmente, prevenir situaciones de victimización en el aula (Cava, 2011).

En esta línea, el centro educativo puede explicitar su política educativa de tolerancia cero hacia cualquier tipo de violencia. Para ello es necesaria la regulación de las normas de convivencia y de los procedimientos para la resolución de los conflictos que alteren la convivencia escolar, con protocolos claros y efectivos, conocidos por la comunidad educativa. Solo una buena convivencia escolar puede permitir el desarrollo integral del alumnado, objetivo último de la educación.

\subsection{Revisa el lenguaje y perspectivas de los contenidos curriculares que impartes}

Los libros de texto, folletos, obras literarias, objetos digitales y demás materiales curriculares de tus asignaturas deben ser objeto de tu análisis, con especial atención a la detección de la presencia de posibles prejuicios o estereotipos sexistas y al uso de un lenguaje inclusivo. Por su parte, la Inspección Educativa de las comunidades autónomas que ya han aprobado su plan de igualdad se encarga de supervisar el desarrollo del currículo mínimo por parte de las administraciones educativas de la correspondiente Comunidad Autónoma.

En los contenidos de las diversas materias, puedes compensar la ausencia secular de las mujeres, recuperando sus obras, su presencia y sus logros (Simón, 2011). Dice Foucault (1997) que lo que no tiene nombre no existe. Asimismo, lo que no se dice no siempre es obvio (Authier-Revuz, 1995). Así pues, "la institución educativa es la encargada de transmitir un arbitrario cultural, el cual se ha correspondido, básicamente, con la cultura masculina. Pues bien, esta situación ha de ser modificada con objeto de introducir elementos propios de la cultura femenina" (Rodríguez, 2003: 60). Es también labor del cuerpo docente, no solo de la historiografía que se queda en los libros, el rescatar la historia, las voces y la cultura propiamente femeninas con objeto de hacerlas visibles en la escuela.

Asimismo, en áreas y materias nuevas podemos introducir la educación emocional y los valores de corresponsabilidad, autonomía personal, respeto activo a las diferencias como positivas y deseables, y rechazo de la violencia como solución a los conflictos, creando actitudes cooperativas, respetuosas y solidarias (Simón, 2011). 
Por último, se recomienda incluir en el currículo escolar una "educación para la igualdad" que, además, se plasme de forma transversal en todas las áreas y en el proyecto educativo (Gregori, 2007).

\section{Conclusiones}

Tras una presentación y análisis tanto de la violencia de género en las aulas como de su prevención, nuestra aportación concreta una línea de actuación que debe ser tenida en cuenta en las escuelas, aun sabiendo que no puede implementarse aisladamente, sin atención integral al problema y sin la colaboración de las familias y el entorno, pues ni colegios e institutos son las únicas instituciones educativas ni las relaciones interpersonales acaban donde los muros de los centros.

Sin embargo, dado que las acciones violentas suelen ser abordadas como actos y mediante actos, nos parece relevante insistir en la importancia de la palabra y de los actos de habla en la prevención de las mismas, pues también forman parte de su causalidad y casuística. Así pues, condensamos la idea en diez pautas verbales para ser consideradas por la labor docente: conocer al alumnado y velar por sus derechos verbales; cuidar el lenguaje; dar retroalimentación verbal tras un conflicto y fomentar el diálogo también en otras situaciones; primar la comunicación inmediata del altercado, colaborar con los registros de los mismos, así como en la redacción y cumplimiento de un proyecto educativo de centro igualitario; educar mediante las sinergias de disciplinas artísticas, mediante nuevas metodologías y mediante la selección de contenidos.

\section{BIBLIOGRAFÍA}

- Álvarez, Pilar (2016a): “Así funciona un colegio antiacoso”. En: El País, 1 de febrero, [en línea] Disponible en http://politica.elpais.com/politica/2016/01/29/actualidad/1454084062_497805.html [23/02/2016].

- Álvarez, Pilar (2016b): “Tres docentes serán juzgadas por tolerar un caso de acoso escolar”. En: El Pais, 16 de febrero, [en línea] Disponible en http://politica.elpais.com/politica/2016/02/15/actualidad/1455567426_363152.html [23/02/2016].

- Álvarez, Pilar (2016c): "Uno de cada 10 alumnos asegura que ha sufrido acoso escolar". En: El País, 19 de febrero, [en línea] Disponible en http://politica.elpais.com/politica/2016/02/18/actualidad/1455799539_145548.html [23/02/2016].

- Aroca, Concepción, Ros, Concepción, y Varela, Cristina (2016): "Programa para el contexto escolar de prevención de violencia en parejas adolescentes”. En: Educar, vol. 52, nº. 1, pp. 11-31, [en línea] Disponible en doi: http://dx.doi.org/10.5565/rev/educar.673 [31/01/2016].

- Authier-Revuz, Jacqueline (1995): Ces mots qui ne vont pas de soi. Boucles reflexives et noncoïncidences du dire. Paris: Larousse. 
- Bandrés, Mercedes (2009): "Estudio de casos de abuso sexual infanto-juvenil entre iguales: aportaciones al ámbito clínico y jurídico con menores agresores y víctimas". En: Anuario de justicia de menores, $\mathrm{n}^{\circ} .9$, pp. 154-182.

- Barroso, F. Javier (2016): “Mamá, le están haciendo pupa”. En: El País, 14 de febrero, [en línea] Disponible en http://ccaa.elpais.com/ccaa/2016/02/12/madrid/1455309839_193487.html [23/02/2016].

- Bermúdez, Silvia (2015): "Feminismo y responsabilidad social en el siglo XXI: Evaluaciones en torno a la violencia sexista en la España Constitucional". En: Letras Femeninas, vol. 41, $\mathrm{n}^{\mathrm{o}}$. 1 [número especial], pp. 147-162.

- Blázquez, Macarena, Moreno, Juan Manuel, y García-Baamonde, M. ${ }^{a}$ Elena (2009): “Inteligencia emocional como alternativa para la prevención del maltrato psicológico en la pareja". En: Anales de Psicología, vol. 25, nº. 2, pp. 250-260.

- Butler, Judith (2005): Dar cuenta de sí mismo. Violencia, ética y responsabilidad. Buenos Aires: Amorrortu.

- Cabrera, Yolanda, y Martínez-Bello, Vladimir (2014): “'Libros para niñas y libros para niños': Presencia de estereotipos de género en una colección de libros para dibujar". En: Cuestiones de género: de la igualdad y la diferencia, $\mathrm{n}^{\circ}$. 9, pp. 182-215, [en línea] Disponible en doi: http://dx.doi.org/10.18002/cg.v0i9.1011 [20/02/2016].

- Cava, M. Jesús (2011): "Familia, profesorado e iguales: claves para el apoyo a las víctimas de acoso escolar". En: Psychosocial Intervention, vol. 20, $\mathrm{n}^{\circ} .2,183-192$.

- Contreras, Paola, y Trujillo, Macarena (2014): "Coeducación para la equidad: A propósito del corpus curricular de la educación chilena. Análisis desde una perspectiva de género". En: Cuestiones de género: de la igualdad y la diferencia, $\mathrm{n}^{\circ}$. 9, pp. 29-49, [en línea] Disponible en doi: http://dx.doi.org/10.18002/cg.v0i9.1042 [25/02/2016].

- De la Encarnación, Ana María (2015): "Glosario de términos vinculados al discurso del logro de la igualdad". En Ruth Abril (dir.), Mujer, participación politica y violencia. Barcelona: Huygens, pp. 441-557.

- Díaz-Aguado, María José (2002): Prevenir la violencia contra las mujeres: construyendo la igualdad. Madrid: Ministerio de Trabajo y Asuntos Sociales y el Instituto de la Mujer.

- Diego, Carmen, y González, Montserrat (2014): "La educación sexual en la escuela primaria: intento frustrado de los eugenistas". En: Cuestiones de género: de la igualdad y la diferencia, $\mathrm{n}^{\circ} .9$, pp. 158-181, [en línea] Disponible en doi: http://dx.doi.org/10.18002/cg.v0i9.1150 [25/02/2016].

- Félix, Vicente, Godoy, Carmen, y Martínez, Ismael (2008): "Violencia entre iguales: resultados de un estudio descriptivo de la provincia de Valencia". En: Informació Psicologica, n". 94, pp. 3648.

- Fernández-Llebrez, F., y Camas, F. (2012): Cambios y persistencias en la igualdad de género de los y las jóvenes en España (1990-2010). Madrid: Instituto de la Juventud.

- Foucault, Michel (1997): Las palabras y las cosas. Madrid: Siglo Veintiuno.

- Garrido, Vicente, y Casas, Mar (2009): "La prevención de la violencia en la relación amorosa entre adolescentes a través del taller 'La máscara del amor' ”. En: Revista de Educación, no. 349, pp. 335-360, [en línea] Disponible en: http://www.revistaeducacion.mec.es/re349/re349_16.pdf [23/02/2016].

- González-Gil, Francisca, y Martín-Pastor, Elena (2014): "Educación para todos: formación docente, género y atención a la diversidad". En: Cuestiones de género: de la igualdad y la 
diferencia, $\mathrm{n}^{\circ}$. 9, pp. 11-28, [en línea] Disponible en doi: http://dx.doi.org/10.18002/cg.v0i9.1151 [25/02/2016].

- Gregori, Pilar (2007): “Algo más para avanzar en coeducación”. En: Revista El Clarión, no. 17, pp. 8-9.

- Guijarro, Juan Ramón (2007): “Análisis de los elementos axiológicos en la normativa española para inglés como lengua extranjera”. En: Cauce, nº. 30, pp. 147-164.

- Hidalgo, María (2016): "El método finlandés para acabar con el acoso escolar y ciberbullying que está revolucionando Europa", [en línea] Disponible en http://muhimu.es/educacion/kiva-acoso/\# [31/01/2016].

- Hidalgo-Marí, Tatiana (2015): "El resurgir de la mujer fatal en publicidad: La reinvención de un mito". En: Cuestiones de género: de la igualdad y la diferencia, $\mathrm{n}^{\circ}$. 10, pp. 394-418, [en línea] Disponible en doi: http://dx.doi.org/10.18002/cg.v0i10.1519 [20/02/2016].

- Hurtado, Inmaculada, Saneleuterio, Elia, López-García-Torres, Rocío, Juaristi, Elena, GarcíaAlcober, María, y Abril, Ruth (2015): "La agenda oculta del trabajo invisible: aproximación teórica y categorización". En: Cuestiones de género: de la igualdad y la diferencia, $\mathrm{n}^{\circ}$. 10, pp. 105-128, [en línea] Disponible en doi: www.dx.doi.org/10.18002/cg.v0i10.1591[16/02/2016].

- Jenaro, Cristina, Flores, Noelia, y Castaño, Raimundo (2014): “Actitudes hacia la diversidad: El papel del género y de la formación". En: Cuestiones de género: de la igualdad y la diferencia, $\mathrm{n}^{\circ} .9$, pp. 50-62, [en línea] Disponible en doi: http://dx.doi.org/10.18002/cg.v0i9.1148 [25/02/2016].

- Kärnä, Antti, et al. (2013): "Effectiveness of the KiVa antibullying program: Grades 1-3 and 79". In: Journal of Educational Psychology, nº 105, pp. 535-551.

- Kaukiainen, Ari, y Salmivalli, Christina (2009): "KiVa Antibullying Program. Parents Guide". Publication series From Research into Practice, 5. University of Turku/Finnish Ministry of Education and Culture [en línea] Disponible en http://www.kivaprogram.net/parents/ [24/02/2016].

- Ley Orgánica 3/2007, de 22 de marzo, para la Igualdad Efectiva de Mujeres y Hombres. BOE, $\mathrm{n}^{\circ}$. 71, pp. 12611 a 12645.

- Ley Orgánica 8/2013, de 9 de diciembre, para la Mejora de la Calidad Educativa, $B O E$ n $^{\circ}$. 295, pp. 97858 a 97921.

- López-Francés, Inmaculada, y Vázquez, Victoria (2014): "La perspectiva de género y el papel de la Universidad en el siglo XXI”. En: TESI, vol. 15, nº 4, pp. 241-261.

- López-García-Torres, Rocío, y Saneleuterio, Elia (2015): "Violencia física y verbal en la infancia". En Ruth Abril (dir.): Mujer, participación politica y violencia. Barcelona: Huygens, pp. 415-439.

- Mercy, J. et al. (2003): “La violencia juvenil”. En: Etienne G. Krug et al. (eds.): Informe mundial sobre la violencia y la salud. Washington: Organización Panamericana de la Salud, pp. 25-61.

- Montero, Rosa (2016): "Volando hacia la muerte". En: El País, 21 de febrero, [en línea] Disponible en http://elpais.com/elpais/2016/02/16/eps/1455626181_383546.html [23/02/2016].

- Muñoz, María del Mar, y Fragueiro, María Sandra (2013): "Sobre el maltrato entre iguales. Algunas propuestas de intervención". En: Escuela abierta: Revista de Investigación Educativa, $\mathrm{n}^{\circ}$. 16, pp. 35-49, [en línea] Disponible http://www.ceuandalucia.es/escuelaabierta/pdf/articulos_ea16_pdf/mu\%C3\%B1oz\%2035-49.pdf [31/01/2016].

- Naciones Unidas (1993): Declaración sobre la eliminación de la violencia contra la mujer. Resolución de la Asamblea General 48/104 del 20 de diciembre. Disponible en 
http://www.acnur.org/t3/fileadmin/scripts/doc.php?file=t3/fileadmin/Documentos/BDL/2002/1286 [19/05/2016].

- Organización Mundial de la Salud (1996): Informe mundial sobre la violencia y la salud. Génova: OMS.

- Ortega, Rosario (1998): "Víctimas, agresores y espectadores de la violencia". En: Rosario Ortega y col.: La violencia escolar: qué es y cómo abordarla. Sevilla: Consejería de Educación y Ciencia, pp. 37-50.

- Ortega, Rosario, Ortega-Rivera, Javier, y Sánchez, Virginia (2008): "Violencia sexual entre compañeros y violencia en parejas adolescentes". En: International Journal of Psychology and Psichological Therapy, vol. 1, no. 8, pp. 63-72.

- Povedano, Amapola, et al. (2015): "Victimization, loneliness, overt and relational violence at the school from a gender perspective". En: International Journal of Clinical and Health Psychology, vol. 15, n. 1, pp. 44-51.

- Rodemannl, Helena (2015): "Derechos en Conflicto: Una Ley Anti-Piropo en España". En: Cuestiones de género: de la igualdad y la diferencia, $\mathrm{n}^{\circ} .10$, pp. 151-160, [en línea] Disponible en doi: http://dx.doi.org/10.18002/cg.v0i10.1513 [20/02/2016].

- Rodríguez, M. a del Carmen (2003): "La contribución de la escuela al logro de identidades de género no estereotipadas". En: Investigación en la Escuela, $\mathrm{n}^{\circ}$. 50, Monográfico "Mujeres, feminismo y coeducación", pp. 57-66.

- Romito, Patricia y Grassi, Michele (2007): "Does violence affect one gender more than the oder? The mental health impact of violence among male and female university students". En: Social Sciences and Medicine, $\mathrm{n}^{\circ}$. 65, pp. 1222-1234.

- Sánchez, Ellen, Robertson, Trina Reed, Lewis, Carol M., Rosenbluth, Barri, Bohman, Tom, y Casey, David M. (2001): "Preventing bullying and sexual harassment in elementary schools: The Expect Respect model". En: Journal of Emotional Abuse, vol. 2, nº. 2-3, pp. 157-180, [en línea] Disponible en doi: http://dx.doi.org/10.1300/J135v02n02_10 [31/01/2016].

- Saneleuterio, Elia (2010): "Espacio público y territorialización. La imagen de la mujer en la obra de José Hierro". En: María Mercedes González de Sande (cur.): Donne, identità e progresso nelle culture mediterranee. Roma: Aracne, pp. 429-440.

- Sastre, Ana (coord.) (2016): Yo a eso no juego. Bullying y ciberbullying en la infancia. Madrid: Save the Children, [en línea] Disponible en https://www.savethechildren.es/sites/default/files/imce/docs/yo_a_eso_no_juego.pdf [23/02/2016].

- Simón, María Elena (2011): La igualdad también se aprende: Cuestión de coeducación. Madrid: Narcea, 2. ${ }^{a}$ ed.

- Valls, Rosa, et al. (2008): Violencia de género en las universidades españolas. Barcelona: Universitat de Barcelona.

- Verdú, Ana Dolores (2014): "Igualdad y desigualdad de género en los imaginarios sociales del amor de pareja heterosexual". En: Cuestiones de género: de la igualdad y la diferencia, $\mathrm{n}^{\circ}$. 9, pp. 402-427, [en línea] Disponible en doi: http://dx.doi.org/10.18002/cg.v0i9.1014 [25/02/2016].

- VV. AA. (2008): Guía de Coeducación. Síntesis sobre la Educación para la Igualdad de Oportunidades entre Mujeres y Hombres. Madrid: Ministerio de Igualdad.

- VV. AA. (2014): Plan Estratégico de Igualdad de Oportunidades 2014-2016. Madrid: Ministerio de Sanidad, Servicios Sociales e Igualdad. 